\title{
The World Wide Web and Its Potential for Corporate Environmental Communication: A Study into Present Practices in the Australian Minerals Industry
}

\author{
Sumit K. Lodhia ${ }^{1}$. The Australian National University \\ Sumit.Lodhia@anu.edu.au
}

\begin{abstract}
This study considers the use of the web for corporate environmental communication, which involves engagement with stakeholders in addition to environmental disclosure. It is highlighted that the web provides numerous benefits for communication of environmental information. These benefits have been conceptualised through Media Richness Theory and operationalised through a study into the web based environmental communication practice of companies in an environmentally sensitive industry, the Australian Minerals Industry. The findings of this research indicate that currently, the potential of the web is not extensively utilised by companies for environmental communication.
\end{abstract}

Keywords: Web, Communication, Medium, Environmental companies.

\section{INTRODUCTION}

Companies often disclose environmental information to their stakeholders to provide evidence that they are accountable for their activities and the resultant impact on the environment. This process is referred to as corporate environmental reporting. There is presently an abundance of research into companies that disclose

\footnotetext{
${ }^{1}$ Data is available from the author. The author would like to thank Professor Roger Burritt, Professor Shirley Gregor, and Dr. Geoff Frost for their support towards this project. Professor Russell Craig's useful insights on this paper and Ms. Judi Moncur's editorial assistance are also highly appreciated. Many thanks to the attendees of the APIRA 2004 conference in Singapore, where an earlier version of this paper was presented.
} 
environmental information (see for instance, Roberts, 1992, Gray et al, 1995, Deegan and Gordon, 1996, Burritt and Welch, 1997, Neu et al, 1998, Christopher et al, 1998, O'Donovan, 1999,2002, Buhr, 2001, Deegan, 2002).

Environmental disclosure is often accompanied by engagement with stakeholders so that the environmental agenda is reflective of company and stakeholder needs. The increasing importance of stakeholders in contemporary society (see for example, Andriof et al, 2002) suggests that one dimensional reporting has been replaced by communication with a range of stakeholders. Consequently, this paper perceives environmental communication as a process whereby companies engage with stakeholders in order to disclose environmental information to them.

The annual report is the primary corporate communication medium for disseminating information on a company's financial position. It has also been extensively utilised for communicating environmental information (see Gray et al, 1995, Gray and Bebbington, 2001). However, other communication media such as stand-alone environmental reports are now becoming increasingly popular (see for instance, ACCA, 2001, Gray and Bebbington, 2001, Tilt, 2001). Advertisements and brochures may suffice in communicating environmental information (Zeghal and Ahmed, 1990, Tilt, 2001) while news media can be used by the organization as well as third parties to disseminate information on a company's environmental performance, or to report on specific environmental issues of importance such as environmental incidents (see Brown and Deegan, 1998, Deegan et al, 2002). More recently, environmental communication on the web has also emerged as a complement to communication through conventional print media (see Jones et al, 1998, Jones and Walton, 1999, UNEP, 1999, 2001, Adams and Frost, 2004).

At present, there is limited published research into whether the medium could enhance corporate environmental communication. Most studies have merely examined the content of environmental disclosure without considering the potential that the medium could offer for communication (see for instance, Craven and Otsmani, 1999, Williams and Pei, 2000, Patten, 2002, Cormier and Magnan, 2003, Patten and Crampton, 2003, Andrew, 2003, Campbell and Beck, 2004). This study addresses this gap in literature by focusing on a particular medium, the web and highlighting that it has the potential to enhance corporate environmental communication. The web has the ability to provide information in real time, has 
mass communication and interactive capabilities, and provides various tools for succinct presentation and organization of information. This research, then, examines the contemporary use of the web by companies in an environmentally sensitive industry- the Australian minerals industry, and attempts to establish the extent to which the potential of the web is utilised in practice.

To conceptualise the benefits offered by the web for environmental communication purposes, a theory from the information systems domain, entitled Media Richness Theory is utilised. Prior studies on web based environmental communication are then outlined in order to place the current study into a broader context and identify potential gaps in the literature. The fourth section articulates the research design for this study, followed by discussion of the results of this study and appropriate conclusions in subsequent sections.

\section{MEDIA RICHNESS THEORY}

Daft and Lengel $(1984,1986)$ propose the concept of "media richness" whereby usefulness of the medium is referred to as its richness. Therefore, for communication purposes, a "rich medium" is said to be more useful than a "leaner" medium (Daft and Lengel, 1986) because it has the ability to succinctly communicate the underlying message. The authors outline four criteria for assessing the richness of a medium:

- Immediacy

- Multiple Cues

- Language Variety

- Personal Source

Immediacy refers to the ability of the medium to provide timely information. Multiple Cues imply the ability to communicate messages through differing approaches, such as body, language, voice and tones. On the other hand, Language Variety refers to the use of different words to increase understanding, while Personal Source focuses on the ability to express feelings and emotions. 
Sproull (1991) and Valacich et al (1993) have expanded the criteria for media richness to take into account the potential of modern communication technologies. These include:

- Multiple Addressibility

- Externally Recordable

- Computer Processable Memory

- Concurrency

Multiple Addressibility refers to the ability to convey information simultaneously to multiple users. The criterion Externally Recordable relates to the ability of the medium to provide a record of the communication. This involves being able to document (as well as modify) the process of communication. Computer Processable Memory, on the other hand, refers to the organization and manageability of information electronically, such that searches can be undertaken on them. The final criterion, Concurrency relates to the ability of the medium to facilitate interaction with multiple users simultaneously.

According to Media Richness Theory, the "richness" of a particular medium can be determined by relating these criteria to a particular task. These criteria can be applied to web based environmental communication.

\subsection{Benefits of web based environmental communication}

The web has numerous benefits for communication purposes. An important benefit it offers is its timely dimension (see for instance, Lymer, 1997, 1999, Lymer et al, 1999, Ashbaugh et al, 1999, UNEP, 1999, FASB, 2000, Williams and Pei, 2000, Ettredge et al, 2001). Information can be made available in real time.

The mass communication (see for instance, Lymer, 1997, 1999, Ashbaugh et al, 1999, Ettredge et al, 2001) and global reach abilities (see for instance, Lymer, 1999, Lymer et al, 1999, UNEP, 1999) of the web allow information to be accessible to a wide range of stakeholders. The web also facilitates two way interaction and feedback through emails, discussion forums and bulletin boards (see for instance, 
UNEP, 1999, Jones and Walton, 1999, Lymer et al, 1999, FASB, 2000, Williams and Pei, 2000).

Similarly, improved presentation through graphics, animation and multimedia, and efficient organization through hyperlinks, search and tracking facilities (see for instance, Ashbaugh et al, 1999, Lymer et al, 1999, UNEP, 1999, Jones and Walton, 1999, FASB, 2000) is possible through the web. These assist in enhancing the information that is communicated to stakeholders.

\subsection{Media Richness Theory and web based environmental communication}

Media Richness Theory conceptualises the usefulness of the web for communication purposes (see Lodhia, 2004). As mentioned in the previous section, the web provides timely information and allows increased accessibility and interaction with numerous stakeholders, in addition to assisting in the presentation and organization of the information.

Table 1 illustrates the linkage between Media Richness Theory and web based environmental communication. The timeliness and accessibility aspects of web based communication can be related to Media Richness Theory features explicitly. Moreover, it is clear from Table 1 that the interactive capabilities of the web can allow personalization and facilitate simultaneous interaction with multiple stakeholders. Media Richness Theory also helps to classify the presentation and organization benefits of the web into specific features.

\begin{tabular}{ll}
\hline Benefit provided by the web & Media Richness Theory feature(s) \\
\hline Timeliness & Immediacy \\
Interaction & Concurrency, Personal Source, Multiple Addres- \\
& sibility \\
Accessibility & Multiple Addressibility \\
& Multiple Cues, Language Variety, Personal \\
Presentation and Organization & Source, Computer Processable Memory and \\
& Externally Recordable \\
\hline
\end{tabular}

Table 1. The web and Media Richness theory

Table 2 suggests that the application of Media Richness Theory to web based communication illustrates the benefits that the web has for communication purposes over conventional print media. The web is a "richer" medium for communication 
when compared to conventional print media. Using Media Richness Theory as a framework, the potential of the web for environmental communication can be illustrated.

\begin{tabular}{|c|c|c|}
\hline & Print Media & Web \\
\hline Immediacy & Usually historical information. & $\begin{array}{l}\text { Information can be supplied on a } \\
\text { timely basis and updated regularly. }\end{array}$ \\
\hline Multiple Cues & $\begin{array}{l}\text { Limited ways of presenting infor- } \\
\text { mation in hardcopy form. }\end{array}$ & $\begin{array}{l}\text { Presentation flexibility and visibility } \\
\text { can provide multiple cues for disse- } \\
\text { minating information. }\end{array}$ \\
\hline Language Variety & $\begin{array}{l}\text { Restricted to a general report, with } \\
\text { limited approaches to organizing } \\
\text { information. }\end{array}$ & $\begin{array}{l}\text { Information can be organized through } \\
\text { hyperlinks and menus, increased in- } \\
\text { formation can be provided, different } \\
\text { file formats can be used for down- } \\
\text { loadable information. Integration of } \\
\text { information is also possible through } \\
\text { hyperlinks to another section, page } \\
\text { or website. }\end{array}$ \\
\hline Personal Source & $\begin{array}{l}\text { Information is for a general au- } \\
\text { dience. }\end{array}$ & $\begin{array}{l}\text { Hyperlinks and menus can provide } \\
\text { information for different stakehol- } \\
\text { ders, information can be pushed to } \\
\text { stakeholders through email lists. }\end{array}$ \\
\hline Multiple Addresibility & $\begin{array}{l}\text { Accessibility is often limited due to } \\
\text { geographic barriers, the addresibi- } \\
\text { lity of information may be to the } \\
\text { more "powerful" stakeholders. }\end{array}$ & $\begin{array}{l}\text { Anyone with web access can receive } \\
\text { information due to the global reach } \\
\text { and mass communication potential } \\
\text { of the web. }\end{array}$ \\
\hline Externally Recordable & $\begin{array}{l}\text { Manual ways of tracing the users } \\
\text { of the disclosed information. Past } \\
\text { reports may be provided upon re- } \\
\text { quest. Information is mainly static. }\end{array}$ & $\begin{array}{l}\text { Information can be downloaded and } \\
\text { printed. Log analysis software and } \\
\text { the hit counter facility can be utilized } \\
\text { to keep a record of users of the web- } \\
\text { site. Reports for multiple years can } \\
\text { be archived on a website. Dynamic } \\
\text { nature of websites enables changes } \\
\text { to be made easily. }\end{array}$ \\
\hline $\begin{array}{l}\text { Computer Processable } \\
\text { Memory }\end{array}$ & Manual searching. & $\begin{array}{l}\text { Navigation and management of in- } \\
\text { formation on website is possible } \\
\text { through menus, hyperlinks and sear- } \\
\text { ch engines. Analytical tools can be } \\
\text { utilised. }\end{array}$ \\
\hline Concurrency & $\begin{array}{l}\text { Restricted mechanisms for ob- } \\
\text { taining feedback. Simultaneous } \\
\text { interaction is not possible. }\end{array}$ & $\begin{array}{l}\text { Electronic mechanisms for obtaining } \\
\text { feedback such as emails and auto- } \\
\text { matic feedback forms. Allows two } \\
\text { way interaction through discussion } \\
\text { forums, emails and bulletin boards. }\end{array}$ \\
\hline
\end{tabular}

Table 2. The richness of the web over print media for environmental communication (Adopted from Lodhia, 2004) 


\section{PRIOR STUDIES}

Web based environmental communication studies are a relatively new addition to the environmental communication literature. Most of these studies are based on practices in the UK, US and Australia. Some studies are broader in that they focus on social and environmental communication and these are discussed here with the foresight that environmental issues are a subset of social and environmental (or social responsibility) issues.

The "earlier" literature on web based environmental communication such as Craven and Otsmani (1999), Jones et al (1999), UNEP (1999) and Williams and Pei (2000) indicated that the larger companies and those operating in developed countries are more likely to use the web for environmental communication. More recent literature has focused specifically on larger companies operating in the developed world. These studies include UNEP (2001), Patten (2002), Rikhardsson et al (2002), Cormier and Magnan (2003) Patten and Crampton (2003) and Andrew (2003). The common finding to these studies is that companies are not extensively utilising the web for environmental communication.

Adams and Frost (2004) is to date, the seminal research on web based environmental communication. They analysed the development of the corporate website as a communication medium by companies in Australia, UK and Germany in respect to the corporate reporting of ethical, social and environmental issues coupled with its use for stakeholder engagement. A triangulation of methods was used to collect data; a longitudinal survey of corporate websites, a postal survey, and interviews with personnel of selected companies. Findings suggest that there is a limited use of the web for communication of ethical, social and environmental issues. It is suggested that corporate communication was not the primary objective of the website, there was no strategic consideration of the website as a communication medium and the development of websites was quite restricted. The authors also reveal that the main purpose of developing corporate websites was to raise corporate awareness and to improve corporate image rather than to report ethical, social and environmental issues and use web based technologies to engage stakeholders. They suggest that companies need to increase their content of disclosure through the web, improve accessibility and verification of information presented on websites, and have more sophisticated use of the website for feedback to enable engagement. 
Unermann and Bennett (2004) have added to the limited literature on web based environmental communication by focusing on the interactivity feature of the web. They applied the Habermasian ideal speech situation to Shell's global stakeholder dialogue which was enabled through use of the discussion forum feature of their website.

The authors' contend that while is not possible to have all the conditions for an ideal speech situation to be satisfied in practice, the web does provide the potential to empower stakeholders to engage in democratic debates. Companies can also obtain feedback from stakeholders by utilising mechanisms such as a discussion forum for stakeholder engagement.

A study by Campbell and Beck (2004) is related to social issues but does provide some indication of the extent to which companies are strategically using the web. The authors suggest that in addition to advertising, selling and reporting purposes, companies use the web for reputation management purposes. This assertion is based on the evidence of disclosure through the websites of eight international companies that had allegations of ethical malpractice against them over a number of years.

Prior literature on web based environmental communication can be analysed according to media richness theory criteria. Tables 3 indicates that limited studies have considered the communication potential of the Web. Some of these studies are descriptive, merely focusing on information content on websites and comparing this to print media.

Methods used in content analysis of annual reports are applied to web based disclosure. An attempt has not been made to ascertain the features of the web that could enhance environmental communication. Other studies have addressed only limited communication features of the web. Moreover, existing studies lack theories which could conceptualise web based environmental communication.

This paper addresses these gaps existing in current literature by focusing on web based environmental communication in a specific context. The next section operationalises the various concepts of media richness to environmental communication. 


\begin{tabular}{|c|c|c|c|c|c|c|c|c|c|c|c|c|c|}
\hline 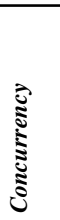 & $\begin{array}{l}n \\
\vdots \\
z \\
\vdots \\
\vdots \\
z \\
z\end{array}$ & 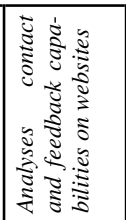 & 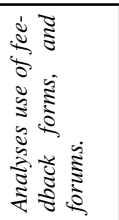 & \begin{tabular}{|l|}
0 \\
2
\end{tabular} & 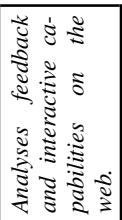 & $\left|\begin{array}{l}0 \\
\vdots \\
\vdots \\
\vdots \\
\vdots \\
z\end{array}\right|$ & 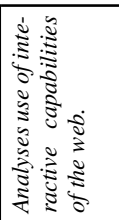 & 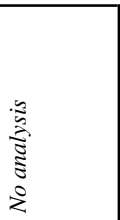 & 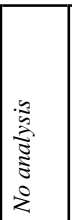 & 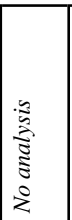 & 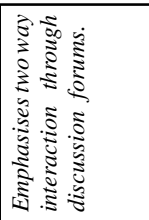 & 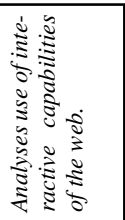 & 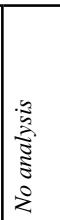 \\
\hline 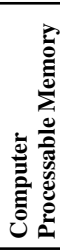 & 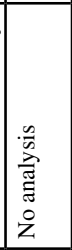 & 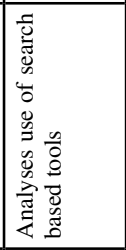 & 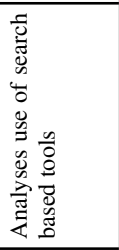 & 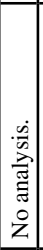 & 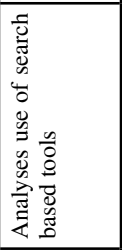 & 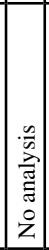 & 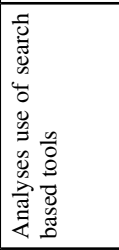 & 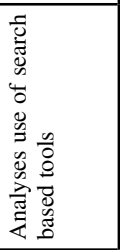 & 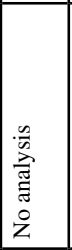 & 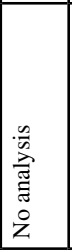 & 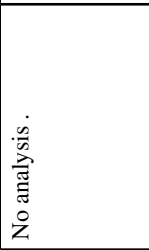 & 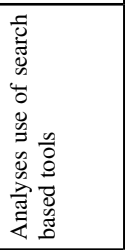 & $\begin{array}{l}\tilde{y} \\
\tilde{z} \\
\tilde{\Xi} \\
\vdots \\
0 \\
z\end{array}$ \\
\hline 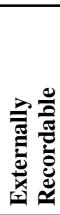 & 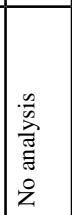 & 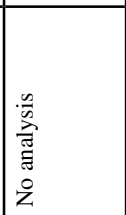 & 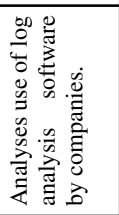 & 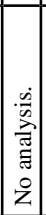 & $\begin{array}{l}\frac{n}{0} \\
\frac{n}{2} \\
\frac{\pi}{\tilde{J}} \\
\vdots \\
0 \\
z\end{array}$ & 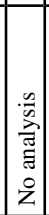 & 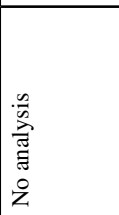 & 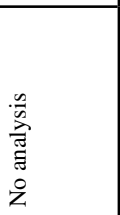 & 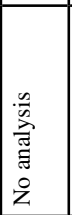 & 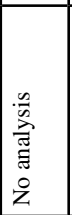 & 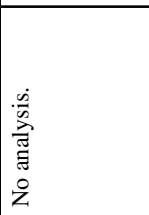 & 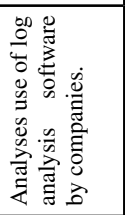 & 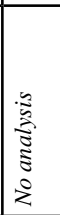 \\
\hline 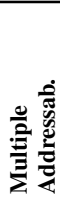 & 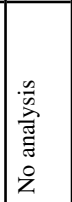 & 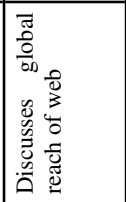 & 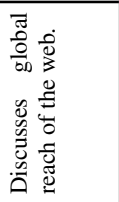 & 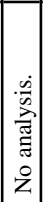 & 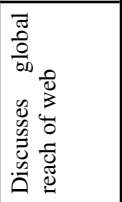 & 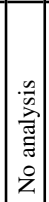 & 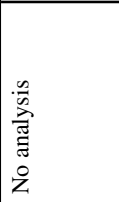 & 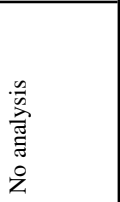 & 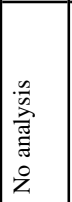 & 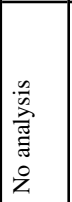 & 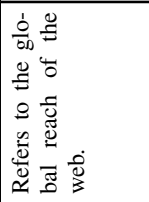 & 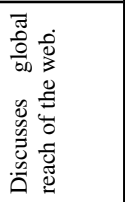 & 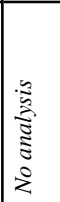 \\
\hline 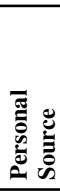 & 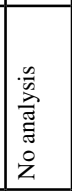 & 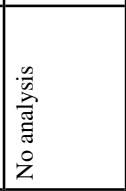 & 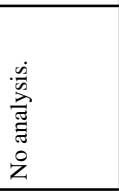 & 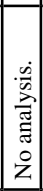 & 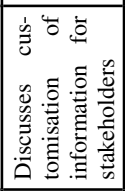 & 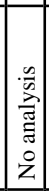 & 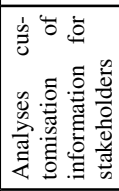 & 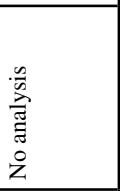 & 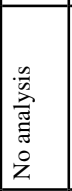 & 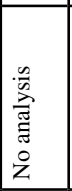 & 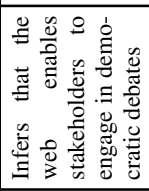 & 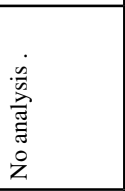 & 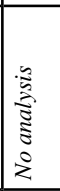 \\
\hline 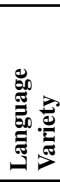 & 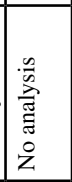 & 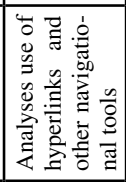 & 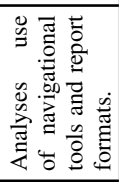 & 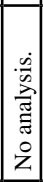 & 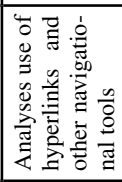 & 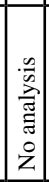 & 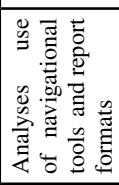 & 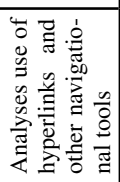 & 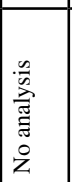 & 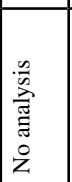 & 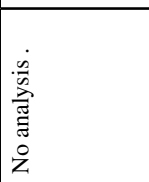 & 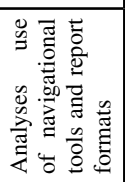 & 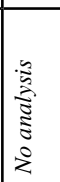 \\
\hline 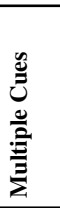 & 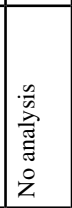 & 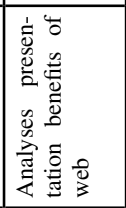 & 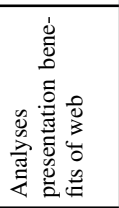 & 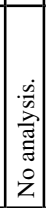 & 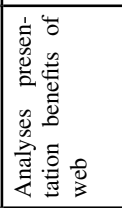 & 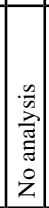 & 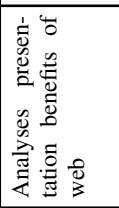 & 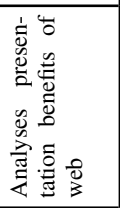 & 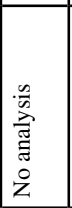 & 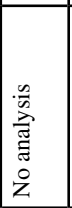 & 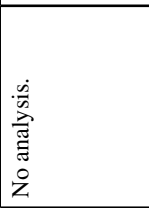 & 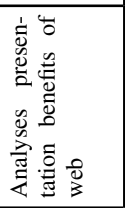 & 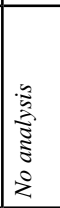 \\
\hline 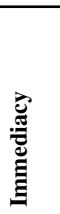 & 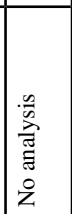 & 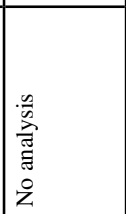 & 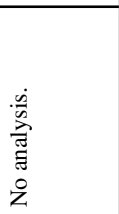 & 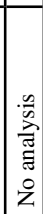 & 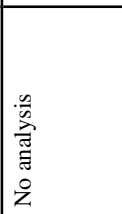 & 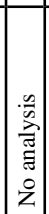 & 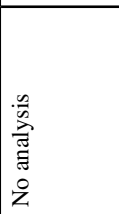 & 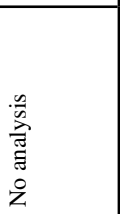 & 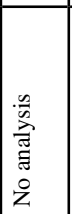 & 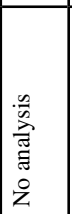 & 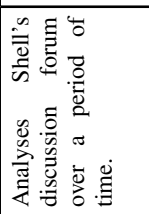 & 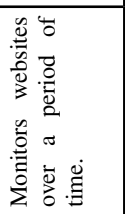 & 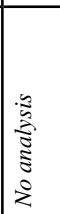 \\
\hline 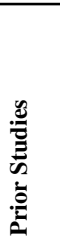 & 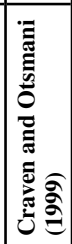 & 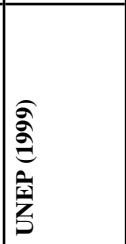 & 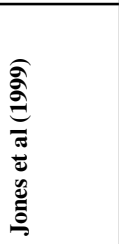 & 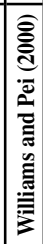 & 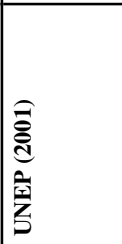 & 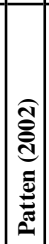 & 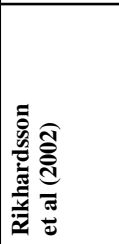 & 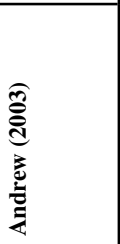 & 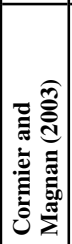 & 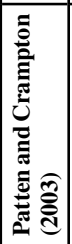 & 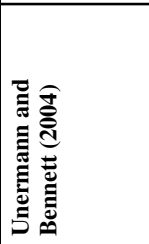 & 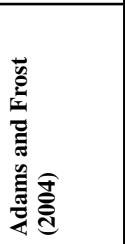 & 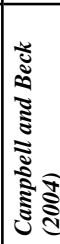 \\
\hline
\end{tabular}

Table 3. Analysis of technological benefits of the web in prior studies 


\section{RESEARCH DESIGN}

This research operationalises the various aspects of Media Richness Theory in relation to corporate environmental communication by establishing a checklist for evaluating the potential offered by the web medium. This is listed and discussed subsequently.

The Immediacy aspect of web based environmental communication can be ascertained through evidence of disclosure of timely information on the web. Such information should also be updated regularly on the webpage. Companies should indicate when particular information on a webpage was updated. An attempt was also made to ascertain whether companies provided some indication of any new environmental information that is placed on websites. Moreover, the study sought to determine whether companies used techniques such as webcasts for their environmental issues. Webcasts are a direct broadcast of an event through the Internet medium.

Multiple Cues in the context of web based environmental communication refers to the extent to which web technology is used to enhance the presentation of information. Graphics, animation and multimedia (audio and video clips) can be utilised to enhance presentation on the web.

Language Variety in relation to web based communication refers to the various ways in which information can be organized. This is facilitated through hyperlinks, menus, and different formats for reports. Hyperlinks can be both internal (within a webpage) and external (to another website). Both summarised and detailed information can be made available through hyperlinks and menus. Furthermore, they can be used to integrate vital information. On the other hand, file formats can include Portable Document Format (PDF), Hypertext Markup Language (HTML) or some other file format. Reports can also be provided in different languages. Web portals, which are a separate website facility for specific reports, can be used to organise environmental information as well. They give stakeholders greater flexibility in viewing their reports.

The Language Variety feature through the use of hyperlinks can also enable a Personal Source for stakeholders. This is possible through having customised information based on the needs of different stakeholders. 
Multiple Addressability refers to the ability to communicate information to multiple stakeholders. Thus, the website should be accessible to all stakeholders and access should not be restricted through passwords or other mechanisms. This enables the mass communication potential of the web to be realized. Email alerts can also be used to target multiple stakeholders, and this adds a Personal Source dimension to web based communication.

Criteria for analysing how the web can be used in practice to communicate environmental information:

\section{Immediacy}

1. Is timely environmental information available i.e. is there regular updating of information?

$\square$ Yes $\quad \square$ No

2. Are details provided on when this information was last updated?

$\square$ Yes $\quad \square$ No

3. Are hyperlinks available to show that new environmental information has been added to the website?

$\square$ Yes $\quad \square$ No

4. Is there evidence to suggest that companies are utilizing webcasts for communicating environmental information?

Yes $\quad \square$ No

\section{Multiple Cues}

5. Which of the following tools are available to improve presentation of environmental information on the website?

Graphics $\square$ Animation $\square$ Multimedia $\square$ Others 


\section{Language Variety}

6. Are the following hyperlinks available:

$\square$ Internal $\square$ External

7. Are pop-up and pull-down menus available?

$\square$ Yes $\quad \square$ No

8. Are hyperlinks/menus available allowing stakeholders to choose the level of detail - summarised versus detailed information?

$\square$ Yes $\quad \square$ No

9. Is there integration of information through key hyperlinks on webpages?

$\square$ Yes $\quad \square$ No

10. Which file format is used for environmental reports?

$\square$ HTML $\square$ PDF $\square$ Other (Specify)

11. Are environmental reports available in different languages?

$\square$ Yes $\quad \square$ No

12. Are environmental reports presented as a web portal?

$\square$ Yes $\quad \square$ No

\section{Language Variety /Personal Source}

13. Are hyperlinks used to provide specific customised information to different stakeholders?

$\square$ Yes $\quad \square$ No 


\section{Multiple Addressibility}

14. Is environmental information accessible to all stakeholders?

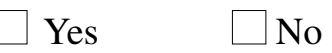

\section{Multiple Addressibility/Personal Source}

15. Are push based mechanisms such as email alerts available?

$\square$ Yes $\quad \square$ No

\section{Externally Recordable}

16. Does the website use facilities like the hit counter and electronic guestbook to track how many stakeholders access environmental information?

$\square$ Yes $\quad \square$ No

17. Is archived information available, such as for example, environmental reports for multiple years?

Yes

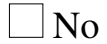

18. Can stakeholders download and print environmental reports?

$\square$ Yes $\quad \square$ No

\section{Computer Processable Memory}

19. Are the following available to assist in navigation of the website

$\square$ Search engines $\square$ Sitemaps $\square$ Menu based Contents list

$\square$ Other navigational tools

20. Are analytical tools available for stakeholders?

$\square$ Yes $\quad \square$ No 


\section{Concurrency}

21. Can stakeholders utilise mechanisms such as automatic feedback forms and electronic surveys?

$\square$ Yes $\quad \square$ No

22. Are any of the following available:

$\square$ Email provisions $\square$ Discussion Forums $\square$ Bulletin Boards

$\square$ Chatrooms

$\square$ Other means to facilitate interaction with stakeholders

The Externally Recordable feature of the web is useful for companies as it allows existing information to be amended easily ${ }^{2}$ and provides them with details on the accessibility of the website by stakeholders.

This is possible through mechanisms such as Hit Counter, Electronic Guestbook or Log Analysis Software ${ }^{3}$. Conversely, stakeholders can also benefit from the availability of archived environmental information and being able to download and print past and current information.

The Computer Processable Memory feature of websites assists in the organization of information. Search engines, site maps and menu based contents lists can be used in this regard. Furthermore, analytical tools can also be used by stakeholders to manipulate information based on their different needs.

Concurrency through the web is facilitated through the use of emails, automatic feedback forms, electronic surveys and two way interaction with stakeholders. Two way interaction is possible through the ability to engage in discussion forums, bulletin boards and chatrooms.

\footnotetext{
2 The amendments made to existing environmental information on websites cannot be assessed thoroughly from the current form of website analysis and requires a longitudinal analysis.

3 The use of log analysis software by companies cannot be determined by merely analysing websites. A need arises to interact with corporate personnel and seek their insights into the use of log analysis software by the company.
} 


\section{DATA COLLECTION}

Having developed the criteria for assessing communication of environmental information, these are related to present web based communication practices in a particular environmentally sensitive industry. The Australian minerals industry is chosen for analysis because companies in this industry have extensive environmental impacts as a result of their operations and extensively communicate environmental information to their stakeholders to provide evidence of their commitment to environmental protection (see, for instance, Christopher et al, 1998, Frost, 1999).

Companies associated with the industry association for the Australian minerals industry, the Minerals Council of Australia, have signed a voluntary code for environmental management ${ }^{4}$. This requires them to improve their environmental performance and report this through various media (Minerals Council of Australia website) $)^{5}$. Past research has also indicated that such companies disclose environmental information to their stakeholders more extensively than their counterparts in the same industry (Christopher et al, 1998). Emphasis in this paper is only on companies associated with the Minerals Council of Australia because its members constitute $85 \%$ of mining companies in Australia. Non- members are very small companies, the majority of which do not even have websites.

Twenty-seven companies were associated with the Minerals Council of Australia as at November 2003, but not all are mining exploration companies. These include a law firm, two accounting firms and several companies that provide mining based services such as mining engineering and supply of specialised mining equipment. Furthermore, there were two companies that are subsidiaries of other companies. One of the mining companies went into receivership during the period of analysis and was eliminated from data collection. Thus, the remaining companies' websites (fourteen companies) were analysed over two different time periods, July and November, 2003. This was undertaken to ensure that any changes made to websites' overtime was taken into account (see Lodhia et al, 2004, for a similar approach in Internet based financial reporting). The approach undertaken

4 This has been expanded to a sustainable development framework more recently.

5 http://www.minerals.org.au/ 
in this study is an initial method for monitoring websites and results did indicate that some changes on websites were noticed within this period of time. However, a more regular monitoring of websites is required in future studies in order to capture the rapidly changing nature of information dissemination on corporate websites 6 . The details of company websites which were analysed are provided in Table 4.

\begin{tabular}{lll}
\hline Company Name & Website address & MNC/Local \\
\hline Anglo Coal Australia Pty Ltd. & www.anglocoal.com.au & MNC \\
AngloGold Australia Ltd. & http://www.anglogold.com.au/default.asp & MNC \\
Barrick Gold of Australia Ltd. & http://www.barrick.com/1_Global_Operations/ & MNC \\
Iluka resources Ltd. & http://www.iluka.com/default.asp & Local \\
Newcrest Mining Ltd. & http://www.newcrest.com.au/ & Local \\
Sons of Gwalia & http://www1.sog.com.au// & Local \\
Ticor Ltd. & http://www.ticor.com.au/home.htm & Local \\
Xtrata Coal Australia Pty Ltd. & http://www.xstrata.com/ & MNC \\
Newmont Mining Corporation & http://www.newmont.com.au/ & MNC \\
Placer Dome Asia Pacific & http://www.placerdome.com/ & MNC \\
Rio Tinto & http://www.riotinto.com/ & MNC \\
Pasminco & http://www.pasminco.com.au/ & Local \\
BHP Billiton Ltd. & http://www.bhpbilliton.com/bb/home/home.jsp & MNC \\
WMC Resources Ltd. & http://www.wmc.com/ & Local \\
\hline
\end{tabular}

Table 4. Company websites analysed in this study

It should also be noted from Table 4 that mining companies in Australia are a mixture of multinational corporations (MNCs) and local companies. There are eight MNCs (approximately $57 \%$ of companies) while the rest are local companies. Only two multinationals have local (Australian) websites.

A maximum of an hour was spent analysing each website over a two week time period in July and November. Most stakeholders would often not spend as much time on websites if information was not available easily to them so the time spent on analysing these websites was considered to be sufficient for analysing websites.

In the discussion of the results of this paper, company names have deliberately not been listed. This is because this initial study has been expanded to include case studies of specific companies. The project assisted in selecting these companies and therefore, it is important that company identities are kept confidential for all the studies.

\footnotetext{
${ }^{6}$ The time constraints associated with this project did not enable continuous monitoring of websites.
} 


\section{RESULTS}

\subsection{General overview}

All fourteen companies had a separate section for environmental issues on their website. This signifies that these companies gave prominence to environmental issues through web based communication.

The titles for the environmental section (or sustainability section) of the company websites can be classified into four categories. These were environment (or environmental management), community and the environment, social (or corporate) responsibility, and sustainability. This suggests that companies were combining environmental information with other types of social information. However, the extent to which environmental information was communicated on websites was far more extensive than other types of social disclosure.

It was observed that in the first round of website analysis, there was a particular local company that had a range of environmental information communicated on its website. However, it was taken over when the second stage of the website analysis took place ${ }^{7}$. Only its archived site existed on the international company's website at that time. This appeared whenever the address for local company's website was typed. The situation persisted for a very brief period of time, and the local company's website did not exist after this. Thus, the extensive range of environmental information communicated on its website was "lost".

As expected, MNCs communicated environmental information on their global operations. However, Australian specific environmental information was not always accessible from some of these websites, highlighting that most MNCs provided aggregated environmental information on their websites.

\subsection{Immediacy}

The immediacy of information on corporate websites was ascertained through an assessment of how recent the information was. For instance, some companies

7 Thus, its details were not included in the website analysis. 
had their most recent environmental reports available or latest press releases replicated on their website.

\begin{tabular}{lcc}
\hline & $N^{\circ}$ of companies & Percentage \\
\hline Timeliness & 7 & 50 \\
Updateability & 0 & 0 \\
New feature & 2 & 14 \\
Webcasts & 6 & 43 \\
\hline
\end{tabular}

Table 5. Immediacy of environmental information on websites

A rather disappointing feature of these websites was the recency of environmental information. Half of the companies did not appear to provide timely information. None of the companies provided an indication of when a particular webpage was updated, even though this is regarded as a critical component in good website design.

Only two websites provided an indication of whether new information has been added to them. Webcasts were used by six of the companies but this was mainly for corporate information. There was not any evidence to suggest that these could be used for environmental issues. However, given that webcasts are related to particular events, it was difficult to ascertain their actual use for environmental issues with this form of website analysis.

\subsection{Multiple cues}

All the companies used graphics on their websites. This mainly included pictures. The use of animated and multimedia tools was limited. Only three companies provided audio or visual enhancements on websites, while animated tools such as applets and flash were used by five of the companies. Thus, companies were not fully utilizing the benefits offered by the web for presentation purposes.

\begin{tabular}{lcc}
\hline & No of companies & Percentage \\
\hline Graphics & 14 & 100 \\
Animation & 5 & 36 \\
Multimedia & 3 & 21 \\
\hline
\end{tabular}

Table 6. Multiple Cues features for environmental information on websites 
The limited use by mining companies of the presentation flexibility offered through the web could be attributed to their consideration of certain stakeholders whose ability to access the website could be restricted through excessive use of graphics. It is assumed that use of certain graphics may impede the access speed of websites, something that could be problematic to some users if their existing bandwidth is slow.

\subsection{Language variety}

Companies used internal hyperlinks and menus quite extensively. External hyperlinks were also provided by five companies. Eight of the companies utilized hyperlinks to distinguish between summarised and detailed information while the same number used hyperlinks to integrate different types of information. A common example was integration of environmental information with other social issues. This suggests that websites were being used in organizing information for all stakeholders.

\begin{tabular}{|c|c|c|}
\hline & No of companies & Percentage \\
\hline Internal hyperlinks & 13 & 93 \\
\hline External hyperlinks & 5 & 36 \\
\hline Menus & 14 & 100 \\
\hline Hyperlinks - general vs. summarised & 8 & 57 \\
\hline Integration through hyperlinks & 8 & 57 \\
\hline File Format - PDF & 13 & 93 \\
\hline Environmental reports in different languages & 2 & 14 \\
\hline Web Portal & 3 & 21 \\
\hline
\end{tabular}

Table 7. Language Variety features used on the Web

PDF was the most common format for environmental reports (sustainability, or health, safety and environment reports were also available) used by thirteen companies while there were three companies that provided a sustainability report web portal. Only two multinational companies provide their reports in more than one language in order to ease the readability of their reports in certain countries where their subsidiaries are located.

Overall, it appeared that in contrast to the limited use of the multiple cues benefits of the web, companies used websites to organize information in a succinct manner and to make the information accessible to stakeholders. 


\subsection{Personal source}

Even though the web has the potential to enable a personal source through the use of pull and push technologies, these were not evident from the websites of most of the mining companies.

\begin{tabular}{lcc}
\hline & No of companies & Percentage \\
\hline Hyperlinks/Menus - Stakeholders & 0 & 0 \\
Email alerts & 7 & 50 \\
\hline
\end{tabular}

Table 8. Personal Source features on websites

Environmental information was not tailored for the needs of different stakeholders through use of hyperlinks or menus. It appeared that most information on websites was generally for all stakeholders and attempts were not made to organize this information based on the needs of specific stakeholders. Conversely, push based mechanisms, such as email alerts were used by half of the companies whose websites were analysed. These were not solely for environmental information.

\subsection{Multiple addressability}

Websites were accessible to all stakeholders. There were no passwords required for access to any type of information. Thus, mass communication of environmental issues could be enabled through the use of websites.

\subsection{Externally recordable}

It was difficult to ascertain whether companies used the web to gain information on the access of the websites by their stakeholders, because hit counter facilities were not available on any of the websites. Similarly, none of the websites had an electronic guest book, while it was not possible to ascertain whether any of these companies were utilising log analysis software.

Stakeholders could download environmental reports from all but one of these websites. The information presented on these websites was printable, with two companies providing printer friendly versions of their webpages. Archived 
information such as for instance, environmental reports from multiple time periods was available for stakeholders on most websites. Ten companies provided this.

\begin{tabular}{lcc}
\hline & No of companies & Percentage \\
\hline Hit Counter & 0 & 0 \\
Electronic Guest Book & 0 & 0 \\
Downloadable reports & 13 & 93 \\
Ease of printing & 14 & 100 \\
Archived information & 10 & 71 \\
Analytical tools & 0 & 0 \\
\hline
\end{tabular}

Table 9. The externally recordable feature on websites

The discussion here reinforces the fact that a lot of corporate emphasis was placed on organizing information on websites and making this accessible to various stakeholders. However, evidence of mechanisms used to trace and manage the stakeholders utilising the web for environmental communication was limited.

\subsection{Computer processable memory}

Computer Processable benefits of the web were focused on search facilities rather than its analytical capabilities. Menu based contents were provided by all the companies. Search engines (twelve companies), followed by sitemaps (seven companies) were also popular and these were for the overall website rather than specifically for environmental information.

\begin{tabular}{lcc}
\hline \multicolumn{1}{c}{ Feature } & No of companies & Percentage \\
\hline Search engines & 12 & 86 \\
Site maps & 7 & 50 \\
Menu based contents & 14 & 100 \\
Analytical Tools & 0 & 0 \\
\hline
\end{tabular}

Table 10. Computer Processable Memory features on websites

Analytical tools were not utilised by any of the companies. Thus, stakeholders did not have the option to manipulate the environmental information based on their specific needs. 


\subsection{Concurrency}

Automatic feedback forms were used by half of these companies but most sought feedback on the website or corporate issues rather than specifically for environmental issues. Only one company sought feedback directly for environmental issues, while three companies sought feedback on their environmental/sustainability reports through their website. This suggests that there were some options available for individual stakeholders to contact the company but these were not always in the context of environmental issues.

Conversely, electronic surveys were not evident on websites. However, surveys are often carried out on an ad-hoc basis and therefore, the general website analysis here was limited in discovering specific trends in web based environmental communication.

\begin{tabular}{lcc}
\hline & No of companies & Percentage \\
\hline Automatic Feedback Forms & 7 & 50 \\
Electronic Surveys & 0 & 0 \\
Email provisions & 12 & 86 \\
Discussion forums/bulletin & 0 & 0 \\
Boards/Chat rooms & & \\
\hline
\end{tabular}

Table 11. Concurrency features on the web

Two-way interaction was only possible through email provisions, which was provided by twelve companies. Again, these were not necessarily in the context of environmental information. Only one of the companies provided various email contacts for a range of issues such as sustainability and financial matters.

None of these companies were utilising discussion forums, bulletin boards or chat rooms. Thus, web technology was not used extensively to promote two-way interaction between a company and its stakeholders.

It appears from the above discussion that companies did have some features available on their websites for enabling engagement with stakeholders but these were generalised in nature. Nevertheless, the potential for stakeholder engagement through the use of the Web did exist. 


\section{SUMMARY, CONTRIBUTIONS AND IMPLICATIONS FOR FUTURE RESEARCH}

This paper has argued that timeliness, mass communication, interactive, and presentation and organisation benefits provided by the web can enhance the corporate environmental communication process. These benefits were conceptualised through Media Richness Theory and applied in the context of web based environmental communication practices of companies in the Australian Minerals Industry. Results highlight that companies are not utilising the potential of the web for communicating environmental information. There is limited use of the Immediacy and Concurrency abilities of the web. The Multiple Cues and Personal Source benefits offered by the Web were also not used extensively by all companies in the Australian minerals industry. Companies were focusing primarily on organizing environmental information through their websites through the Language Variety, Externally Recordable and Computer Processable Memory features and making this accessible to their numerous stakeholders through the Multiple Addressability benefit. The analytical (Computer Processable Memory) and stakeholder tracing (Externally Recordable) capabilities offered by web technologies were also not evident from the general website analysis. Moreover, it was clear from this analysis that companies were providing general features for websites, rather than having specific features for individual sections of websites.

Companies may not be aware of the potential benefits offered by the web for communication purposes. Web based environmental communication could be a learning process for most companies in the absence of guidelines for such a form of communication. On the contrary, companies may be reluctant to use the web for communicating environmental information. This may be due to issues such as a lack of accessibility with certain stakeholders, confidentiality and security issues, the concern over information overload, or even costs associated with website maintenance. Moreover, the use of the web for environmental communication could merely be an attempt by corporations to enhance their legitimacy through public relations exercises. These explanations warrant further investigation and would require moving beyond analysis of websites and interacting with a company's key decision makers (see for example, Adams and Frost, 2004). Nevertheless, exploratory works such as the current study are insightful because they provide 
an initial understanding of contemporary practices in environmentally sensitive industries.

Whilst utilizing the corporate website for providing information to a range of stakeholders is applauded, companies can make extensive use of web technology. The Web has emerged in leaps and bounds over the years, and while it has extensive usage for e-commerce activities, corporate communication is another area where it could be successfully utilized. Continuous communication, use of presentation features (such as audio and video clips, webcasts, and even video conferencing), greater interactivity and personalization are envisaged for web based environmental communication in the future. Increased academic research in this area and development of methods that could capture the dynamic nature of change of information on websites will also be beneficial.

This study has contributed theoretically by outlining a theory that conceptualises the benefits offered by the web. The framework advanced by this research is not merely restricted to environmental information; it can be used to analyse disclosure of other types of information on the web. Similarly, the research design for this study can be applied in other contexts.

This study has highlighted that there are differences in analysing information on websites when compared to print media. Accordingly, websites should be analysed at different periods rather than being analysed at one particular time. It is also important to consider the potential benefits offered by the web when studying the process of environmental communication through this medium. Most prior studies have merely applied to web based environmental communication research the content analysis techniques that have been commonly used in studies on corporate environmental reporting through conventional print media. "New" methods for analysing environmental information on corporate websites have been developed in this study, providing valuable insights for future studies in this area.

In addition to the contributions to theory and research design, this study has also expanded the literature on web based environmental communication by focusing on current practices in an environmentally sensitive industry (the Australian minerals industry)where environmental communication is vital for companies (IIED, 2002, Peck and Sinding, 2003). The results indicate that the situation in a particular 
industry in relation to web based environmental communication is similar to the general findings of Adams and Frost (2004). This provides further support to the premise that the communication potential of the web is not being fully utilised by many companies (Ibid), even those in environmentally sensitive industries.

In relation to practice, this study highlights the communication potential of the web for environmental communication purposes and suggests that companies are not fully utilising this potential for environmental communication. It is suggested that policy makers such regulators and industry associations could play a key role in providing an impetus to companies to move beyond merely using websites for organizing environmental information and to utilise the "full" communication potential of the web.

The web can be used to improve the process of corporate environmental communication and mandatory and voluntary initiatives are needed to encourage this practice. This could lead to an enhanced form of environmental communication, providing timely, accessible, and well presented and organised information to stakeholders who could also interact on a more regular basis with companies. Moreover, broader policy issues such as security and authentication of information on websites, and overcoming the digital divide will need to be dealt with in order to enable the process of web based environmental communication.

Future research should complement analysis of corporate websites with the more traditional research methods, such as surveys and interviews, which could seek further information on the processes involved in web environmental communication, as well as explain the reasoning behind the status of present practices. There is a need for more studies which use surveys and interviews to complement website analysis. For instance, interviews could be held with mining practitioners to comprehend the findings of this research and seek their views on web based environmental communication. Stakeholder views on this could also be obtained. An analysis of web based environmental communication practices in other industries could be undertaken to establish whether the existing findings from the literature on the limited use of the web for environmental communication also applies in other situations. These will provide a richer description of the different contexts in which the web is used for environmental communication. 


\section{REFERENCES}

ACCA (2001): An Introduction to Environmental Reporting The Certified Accountants Educational Trust, London

ADAMS, C.A.; FROST, G.R. (2004): The Development of the Corporate Website and Implications for Ethical, Social and Environmental Reporting through these Media, The Institute of Chartered Accountants of Scotland, Edinburgh.

ANDREW, J. (2003): "Corporate Governance, the Environment, and the Internet" Electronic Green Journal, vol. 19, http://egj.lib.uidaho.edu/egj19/andrew1.html

ANDRIOF, J.; WADDOCK, S.; HUSTED, B.; SUTHERLAND RAHMAN, S. (Eds), (2002a): Unfolding Stakeholder Thinking, Greanleaf Publishing Limited, Sheffield.

ASHBAUGH, H.; JOHNSTONE, K.M.; WARFIELD, T.D. (1999): "Corporate Reporting on the Internet”, Accounting Horizons, vol. 13, n. 3:241-257.

BROWN, N.; DEEGAN, C. (1998):“The public disclosure of environmental performance information - a dual test of media agenda setting theory and legitimacy theory", Accounting and Business Research, vol. 29, n. 1:21-41.

BUHR, N. (2001): "Corporate Silence: Environmental Disclosure and the North American Free Trade Agreement", Critical Perspectives on Accounting, vol. 12: 405-421

BURRITT, R.L.; WELCH, S. (1997): “Accountability for Environmental Performance of the Australian Commonwealth Public Sector", Accounting, Auditing, and Accountability Journal, vol. 10, n. 4: 532-561

CAMPBELL, D.J.; BECK, C (2004): "Answering allegations: the use of the corporate website for issue-specific reputation management", Business Ethics: A European Review vol. 13, n. 2/3: 100-116

CHRISTOPHER, T.; CULLEN, L.; SOUTAR, G (1998): “Australian Mining Companies Environmental Disclosure", Accountability and Performance, vol. 4, n. 2: $17-41$. 
CORMIER; MAGNAN (2003): "The impact of the web on information and communication modes: the case of corporate environmental disclosure", International Journal of Technology Management, vol. 27, n. 4 : 20-34

CRAVEN, B.M.; OTSMANI, B. (1999): "Social and environmental communication on the Internet by leading UK companies", Paper presented at the European Accounting Association (EAA) Annual Conference, Birmingham

DAFT, R.; LENGEL, R. (1984): "Information Richness: A New Approach to Managerial Behaviour and Organization Design”, Research in Organizational Behaviour vol. 6:191- 233.

DAFT, R.; LENGEL, R. (1986): "Organizational Information Requirements, Media Richness and Structural Design”, Management Science, vol. 32 n. 5:554- 571.

DEEGAN, C. (2002): "Introduction: The legitimising effect of social and environmental disclosures - a theoretical foundation", Accounting, Auditing, and Accountability Journal, vol. 15, n. 3 :282-311

DEEGAN, C.; RANKIN, M.; TOBIN, J. (2002): "An examination of the corporate social and environmental disclosures of BHP from 1983-1997: A test of legitimacy theory", Accounting, Auditing, and Accountability Journal, vol. 15, n. 3:312-343

ETTREDGE, M.; RICHARDSON, V.J.; SCHOLZ, S. (2001): "The presentation of financial information at corporate Web sites", International Journal of Accounting Information Systems, vol. 2:149-168

FINANCIAL ACCOUNTING STANDARDS BOARD (FASB) (2000): Business Reporting Research Project: Electronic Distribution of Business Reporting Information Steering Committee Report Series http://www.fasb.org

FROST, G.R (1999): "Environmental reporting : An analysis of company annual reports of the Australian Extractive Industries 1985-1994" Unpublished PhD dissertation, University of New England.

GRAY, R.H.; BEBBINGTON, K. J. (2001): Accounting for the environment, $2^{\text {nd }}$ edition Sage London. 
GRAY, R.H.; KOUHY, R.; LAVERS, S. (1995): “Corporate Social and Environment reporting: a review of the literature and a longitudinal study of UK Disclosure", Accounting, Auditing, and Accountability Journal, vol. 8, n. 2: 47-77

INTERNATIONAL INSTITUTE FOR ENVIRONMENT AND DEVELOPMENT (IIED) (2002): Facing the Future: The report of the Mining Minerals and Sustainable Development (MMSD) Australia project Australian Minerals and Energy Foundation (AMEEF), Australia.

JONES, K.; ALABASTER, T.; HETHERINGTON, K. (1998): "Virtual environments for environmental reporting", Greener Management International vol. 21, Spring,

JONES, K.; ALABASTER, T.; HETHERINGTON, K. (1999): “Internet-Based Environmental Reporting: Current Trends", Greener Management International vol. 26:69-90.

JONES, K.; WALTON, J. (1999): "Internet-Based Environmental Reporting: Key Components" in Bennett, M, James, P and Klinkers, L eds, Sustainable Measures: Evaluation and Reporting of Environmental and Social Performance Greenleaf Publishing UK.

LODHIA, S. (2004): “Corporate Environmental Reporting Media: A Case for the World Wide Web", Electronic Green Journal, vol. 20 http://egj.lib.uidaho. edu/egj20/lodhia1.html

LODHIA, S.; ALLAM, A.A.; AND LYMER, A. (2004): “Corporate Reporting on the Internet in Australia: An Exploratory Study", Australian Accounting Review vol. 14, n. 3:64-71.

LYMER, A.M (1997): “The use of the Internet for corporate reporting - a discussion of the issues and survey of current usage in the UK", Journal of Financial Information Systems http://www.shu.ac.uk/schools/fsl/fisjnl//vol1996/pprs1997/ lymer97.htm

LYMER, A.M. (1999): "The Internet and the future of corporate reporting in Europe", The European Accounting Review, vol. 8, n. 2: 289-301. 
LYMER, A.; DEBRECENY, R.; GRAY, G.L.; RAHMAN, A (1999): Business Reporting on the Internet, A Report Prepared for the International Accounting Standards Committee, IASC, London.

NEU, D.; PEDLEY, R.V.; WARSAME, H. (1998): "Managing Public Impressions: Environmental Disclosures in Annual Reports", Accounting Organizations and Society, vol. 23, n. 3:265- 282

O'DONOVAN, G. (1999): “Managing legitimacy through increased corporate environmental reporting: An exploratory study", Interdisciplinary Environmental Review, vol. 1, n. 1: 63-97

O'DONOVAN, G. (2002): "Environmental Disclosures in the annual report: extending the applicability and predictive power of legitimacy theory", Accounting, Auditing, and Accountability Journal, vol. 15, n. 3 :344-371

PATTEN (2002): "Give or Take on the Internet: An Examination of the Disclosure Practices of Insurance Firm Web Innovators", Journal of Business Ethics vol. 36, n. 3: 247-259.

PATTEN, D.; CRAMPTON, W. (2003): "Legitimacy and the Internet: An examination of corporate web page environmental disclosures", Advances in Environmental Accounting and Management vol. 2: 31-57

PECK; SINDING (2003): "Environmental and Social Disclosure and Data Richness in the Mining Industry", Business Strategy and the Environment vol. 12:131-146

RIKHARDSSON, P.; ANDERSON, A.; BANG, H (2002): "Sustainability reporting on the internet: a study of the Global Fortune 500", Greener Management Inetrnational vol. 40: 57-75.

ROBERTS, R.W. (1992): "Determinants of Corporate Social Responsibility Disclosure: An Application of Stakeholder Theory", Accounting Organizations and Society, vol. 17, n. 6:.595-612.

SPROULL, R. (1991): “A lesson in electronic mail”, In , L. Sproull and S. Kiesler ed. Connections: New ways of working in the networked organization : 177-184 MIT Press. 
TILT, C.A (2001): "Environmental disclosures by Australian Companies: what is happening outside the annual report", Proceedings of Asia Pacific Interdisciplinary Research in Accounting (APIRA) Conference.

UNEP (1999): The Internet Reporting Report Engaging Stakeholder Series UNEP/Sustainability.

UNEP (2001): Virtual Sustainability Engaging Stakeholder Series UNEP/ Sustainability.

UNERMAN, J.; BENNETT, M. (2004): "Increased stakeholder dialogue and the Internet: towards greater corporate accountability or reinforcing capitalist hegemony?", Accounting, Organisations and Society vol. 29, n. 7. 685-707.

VALACICH, J.; PARANKA, D.; NUNAMAKER, J. (1993): "Communication Concurrency and the New Media", Communication Research, vol. 20, n. 2: 249276.

WILLIAMS, S.M.; PEI, C.H.W. (2000): "Corporate Social Disclosure by Listed companies on their web sites: An international comparison", International Journal of Accounting, vol. 34, n. 3: 389-419

ZEGHAL, D.; AHMED, S.A. (1990): "Comparison of Social Responsibility Information Disclosure Media Used by Canadian Firms", Accounting, Auditing, and Accountability Journal, vol. 3, n. 1: 38-53. 